\title{
In Vitro Antioxidant Potentials of Traditional Chinese Medicine, Shengmai San and Their Relation to in Vivo Protective Effect on Cerebral Oxidative Damage in Rats
}

\author{
Haruyo ICHIKAWA and Tetsuya KonISHI* \\ Department of Radiochemistry-Biophysics, Niigata University of Pharmacy and Applied Life Science; 5-13-2 Kamishin- \\ ei, Niigata 950-2081, Japan. Received February 6, 2002; accepted April 5, 2002
}

\begin{abstract}
The preventive effects of Shengmai San (SMS), a traditional Chinese herbal medicine (TCM), was studied on cerebral ischemia-reperfusion injury in rats as a model of antioxidant-based composite therapy. Two biochemical indicators of oxidative damage, thiobarbituric acid reactive substance (TBARS) formation and glutathione peroxidase (GPX) loss were measured in the brain after forebrain ischemia-reperfusion treatment and both were inhibited in all rats administered SMS (15 g original herbs $/ \mathrm{kg}) 2 \mathrm{~h}$ before the ischemia-reperfusion. Histochemical study of the brain slice using TTC staining revealed that the SMS effectively reduced infarct area caused by the cerebral ischemia-reperfusion. The antioxidant potentials of SMS preparations were determined in vitro by five different assay methods and were related to the in vivo effectiveness of SMS in protection against brain damage. Inhibitory effect on TBARS formation in vivo showed better correlation with superoxide radical scavenging and DPPH quenching activity in vitro rather than with the other in vitro antioxidant indicators. On the other hand, the in vivo prevention of GPX activity loss showed better correlation with in vitro crocin bleaching inhibition than with the other in vitro antioxidant indicators. It was also suggested that the in vitro TBARS inhibitory activty of SMS is not a good indication to predict the in vivo effectiveness of SMS on inhibition of either TBARS formation or GPX activity loss.
\end{abstract}

Key words traditional Chinese medicine; Shengmai San; cerebral ischemia-reperfusion; antioxidant activity

Reactive oxygen species (ROS) are related to complex pathophysiological conditions such as lifestyle- and/or agerelated diseases. ${ }^{1,2)}$ In particular, the brain is quite susceptible to oxidative insult due to the low levels of physiological antioxidants and antioxidant enzymes in the brain. ${ }^{3)}$ In fact, ROS have been implicated in brain neurodegenerative diseases such as Alzheimer's and Parkinson's diseases. ${ }^{4)}$ Although ROS are instrumental in the initiation of lipid peroxidation, many other reactions such as cytoplasmic $\mathrm{Ca}^{2+}$ release ${ }^{5)}$ also play crucial roles in the subsequent propagation of tissue injury. Thus, antioxidant protection of the brain against oxidative stress cannot be successfully achieved by the use of radical scavenging antioxidant alone. Indeed, several trials with small molecular antioxidants have been done but results were not enough satisfactly. ${ }^{6-13)}$

In this sense, antioxidative traditional Chinese medicine (TCM) is an attractive target for the study as a model of antioxidant-based composite therapy against cerebral oxidative damage, as TCM has been used as an alternative medicine for treating complex pathophysiological conditions, even for the diseases that Western medicine has failed to allocate a specific diagnostic name. ${ }^{14-17)}$

However, the use of TCM is accompanied by difficulties in controlling the quantitative aspect of the treatment or setting up the therapeutic strategy, because the lack of sufficient knowledge about which component is ultimately active and how the component-component interaction affects the overall action. Therefore, some new overall criteria is necessary for evaluating therapeutic potential of mixed formulation such as TCM against complex pathophysiological conditions. Since, oxidative stress has been implicated in many complex conditions to which TCM can be applicable. It is of interest to study how the in vitro antioxidant potential of certain TCM formula relates to the in vivo activity of protecting oxidative tissue damage.
In the present study, we precisely examined the antioxidant potential of a traditional Chinese medicine, Shengmai San (SMS) in vitro and then discussed how the in vitro activity or which indication of the in vitro antioxidant assay relates to the in vivo effectiveness of SMS in preventing cerebral oxidative damage induced by ischemia-reperfusion in rats. SMS is a traditional formulation being used for treating symptoms related to cardiac disorder but was shown to have effective protection against oxidative damage in rat brain in our previous study. ${ }^{18)}$

\section{MATERIALS AND METHODS}

Reagents Constituent herbs of Shengmai San, Panax ginseng and Ophiopogon japonicus and Shisandra chinensis were the products of Korea, Sichuan, P.R. China, Hei Long Liang, P.R. China, respectively. Dimethylpyrroline oxide (DMPO) was purchased from LABOTEC Co., Ltd., Tokyo. $\mathrm{FeSO}_{4}$ was purchased from Kanto Chemical Co, Ltd., Japan. Xanthine oxidase (XOD, $20 \mathrm{U} / \mathrm{ml}$, from cow milk) was purchased from Boehringer Mannheim Co., Germany. Crocin was extracted by diethyl ether from dried saffron purchased from a local supermarket. 6-Hydroxy-2,5,7,8-tetramethylchroman-2-carboxylic acid (Trolox) was purchased from Aldrich Chem. Co. All other reagents, including 2,2'-Azobis (2-amidino-propane) dihydrochloride (AAPH), and diethylene triamine- $N, N, N^{\prime}, N^{\prime \prime}, N^{\prime \prime}$-penta acetic acid (DTPA) were purchased from Wako Pure Chemical Industries Co., Ltd., Japan. BCA protein assay reagent was purchased from Piearce Chemical Co.

SMS Preparation The SMS preparation used in this study was kindly prepared by Kotaro Kampo Co., Ltd., Japan. Briefly, the three component herbs of Shengmai San, Panax ginseng (48g), Ophiopogon japonicus (48 g), and Schisandra chinensis $(24 \mathrm{~g})$ were suspended in $1200 \mathrm{ml}$ dis- 
tilled water, soaked for $1 \mathrm{~h}$, and then decocted for $1 \mathrm{~h}$. The supernatant was filtered through gauze, and the filtrate was then freeze-dried. The dried filtrate was mixed with Neusilin FL2 as an additive to produce a granulated SMS preparation. The SMS granules were stored at $-80^{\circ} \mathrm{C}$ until use. For each experiment, the SMS granules were weighed precisely and solubilized in distilled water to make the final concentrations indicated.

Animal Treatment Male Wister rats (6 weeks old and $160-168 \mathrm{~g}$ body weight) were purchased from SLC Inc., Japan. They were allowed free access to pelleted diet and water before the experiments. Anesthesia was induced by diethyl ether and maintained by pentobarbital. In order to examine the dose-response effect of SMS against cerebral ischemia-reperfusion injury, $2 \mathrm{ml}$ of each SMS solution at different concentrations $(0.15,0.3,0.6,1.2 \mathrm{~g} / \mathrm{ml})$ was administered directly into the lumen of duodenum exposed by abdominal incision $2 \mathrm{~h}$ before the ischemia operation. Cerebral ischemia was produced by occlusion of both the right and left common carotid arteries exposed through a middle skin incision, using aneurysm clips for $85 \mathrm{~min}$. Ischemic condition was confirmed by the change in color of the eyeball from red to white. At the end of the ischemic period, the carotid arteries were declamped to allow blood reperfusion. All rats were decapitated under anesthesia after $45 \mathrm{~min}$ of reperfusion, and then the whole brain was removed for biochemical analysis.

For each experiment outlined below, biochemical measurements were taken for three groups of rats 1: Normal control; rats not given any treatment, 2: Vehicle control; rats administered Neusilin instead of SMS before ischemia followed by reperfusion, 3: SMS treated; rats treated with SMS before cerebral ischemia-reperfusion as outlined above.

Tissue Homogenate The whole brain was removed, weighed, and then chilled in ice-cold saline. After washing with $0.85 \% \mathrm{NaCl}$, the tissue was suspended in cold $0.05 \mathrm{M}$ phosphate buffer containing $1.15 \%(\mathrm{w} / \mathrm{v}) \mathrm{KCl}(9 \mathrm{ml}$ per $1 \mathrm{~g}$ wet tissue), and then homogenized at $0{ }^{\circ} \mathrm{C}$ using an ULTRA TRAX homogenizer.

Protein concentration of the homogenate was determined by BCA protein assay using bovine serum albumin as a standard (PIERCE).

TTC Staining of Brain Tissue TTC (2,3,5-triphenyltetrazolium chloride) staining of brain slices was performed as previously described. ${ }^{20}$ The brains removed were froze for $10 \mathrm{~min}$ at $-20^{\circ} \mathrm{C}$, and then six coronal brain sections, 6$8 \mathrm{~mm}$ thick, were sliced from the front pole using a razor. The sections were incubated in phosphate buffered saline containing $2 \% \mathrm{TTC}$ at $37^{\circ} \mathrm{C}$ for $10 \mathrm{~min}$, and then kept in $10 \%$ buffered formalin ( $\mathrm{pH} 7.0$ ) overnight.

Thiobarbituric Acid Reactive Substance (TBARS) in Brain Tissue Homogenate TBARS formation was determined as previously reported. ${ }^{21)}$ Briefly, an aliquot of brain homogenate ( $1 \mathrm{mg}$ as protein) was mixed with $0.2 \mathrm{ml}$ of $15 \%$ sodium dodecyl sulfate (SDS), $1.5 \mathrm{ml}$ of $20 \%$ acetic acid and $1.5 \mathrm{ml}$ of $0.8 \% \mathrm{TBA}$, and then the volume was adjusted to $4.0 \mathrm{ml}$ with distilled water. After boiling at $95^{\circ} \mathrm{C}$ for $60 \mathrm{~min}$, the reaction solution was extracted with $n$-buthanol/pyridine $(15: 1 \mathrm{v} / \mathrm{v})$. Absorbance at $532 \mathrm{~nm}$ was determined for the organic layer after centrifugation.

Glutathione Peroxidase (GPX) Activity in Brain Tissue
Homogenate GPX activity was determined according to the method of Albrecht. ${ }^{22)}$ Briefly, an aliquot of brain homogenate $(0.4 \mathrm{mg}$ as protein) in $0.05 \mathrm{M}$ phosphate buffer containing $1.15 \%(\mathrm{w} / \mathrm{v}) \mathrm{KCl}$ was mixed in a quartz cuvette with $935 \mu \mathrm{l}$ of coupling solution (containing $33.6 \mathrm{mg}$ disodium EDTA, $6.5 \mathrm{mg} \mathrm{NaN}_{3}, 30.7 \mathrm{mg}$ of GSH, $16.7 \mathrm{mg} \mathrm{NADPH}$ and 100 units of GSH reductase in $100 \mathrm{ml}$ of $50 \mathrm{~mm}$ Tris $-\mathrm{HCl} \mathrm{pH}$ 7.6) and the volume then adjusted to $975 \mu \mathrm{l}$ with $0.05 \mathrm{M}$ phosphate buffer. Kinetic decay of NADPH fluorescence (Ex. $355 \mathrm{~nm} / \mathrm{Em} .465 \mathrm{~nm}$ ) was measured after the addition of $25 \mu \mathrm{l}$ of $1 \mathrm{mM} \mathrm{H}_{2} \mathrm{O}_{2}$ as the substrate using a Hitachi model 650-60 fluorescence spectrophotometer.

Hydroxyl Radical Scavenging Activity Measurement in Vitro Hydroxyl radical scavenging activity of SMS was determined as reported previously using the Fenton reaction as the hydroxyl radical source. $\left.{ }^{23}\right)$ The reaction mixture $(300 \mu 1$ total volume) containing defined amounts of SMS, $20 \mathrm{~mm}$ DMPO, $10 \mathrm{mM} \mathrm{H}_{2} \mathrm{O}_{2}$, and $0.1 \mathrm{mM} \mathrm{FeSO}_{4}$ was quickly transferred into a $250 \mu \mathrm{l}$ quartz flat cell. Exactly $1 \mathrm{~min}$ after the addition of $\mathrm{FeSO}_{4}$, the electron spin resonance (ESR) spectra of DMPO-OH was determined using a JEOL JES-TE 200 ESR spectrometer (X-band Microwave Unit) with the following settings: microwave power; $8 \mathrm{~mW}$, microwave frequency; $9.18 \mathrm{GHz}$, modulation amplitude; $0.1 \mathrm{~m}$, time constant; $0.03 \mathrm{~s}$, sweep time; $30 \mathrm{~s}$, center fields; $331.6 / 321.6 \mathrm{mT}$.

Superoxide Scavenging Activity Measurement in Vitro Superoxide scavenging activity of SMS was measured according to the methods of Finkelstein et al., ${ }^{24)}$ using the hypoxanthine (HPX)/xanthine oxidase (XOD) system as the superoxide anion radical generator. The reaction mixture (total $300 \mu \mathrm{l}$ ) containing defined amounts of SMS, $1 \mathrm{~mm}$ DTPA, $0.5 \mathrm{~mm}$ hypoxanthine, DMPO and $0.1 \mathrm{U} / \mathrm{ml}$ xanthine oxidase in $0.2 \mathrm{M}$ phosphate buffer ( $\mathrm{pH} 7.8$ ) was quickly transferred into a $250 \mu \mathrm{l}$ quartz flat cell. The ESR spectra of DMPO$\mathrm{OOH}$ was determined exactly $1 \mathrm{~min}$ after the addition of XOD. The ESR settings were the same as for DMPO-OH.

Crocin Bleaching Activity Measurement Crocin extraction and the bleaching test were carried out according to the method of Wolf et al. ${ }^{25)}$ with minor modifications. To $3.0 \mathrm{ml}$ of $0.1 \mathrm{M}$ phosphate buffer $(\mathrm{pH} 7.0)$ containing defined amounts of SMS and $10 \mu \mathrm{M}$ crocin, $75 \mu \mathrm{l}$ of AAPH $(0.5 \mathrm{M}$ stock solution) was added to initiate crocin bleaching at $40{ }^{\circ} \mathrm{C}$. The decrease in the absorbance at $443 \mathrm{~nm}$ was kinetically recorded.

AAPH-Induced Lipid Peroxidation in Vitro AAPH initiated lipid peroxidation was determined in the presence or absence of SMS, using the TBARS method. Briefly, reaction mixture $(300 \mu \mathrm{l})$ containing liver homogenate (1 mg as protein), $0.1 \mathrm{ml}$ AAPH (1 mM aqueous solution) and defined amount of SMS was incubated aerobically at $37^{\circ} \mathrm{C}$ for $30 \mathrm{~min}$. TBARS formation was determined as outlined above. $^{21)}$

DPPH Radical Scavenging Assay in Vitro DPPH (1,1diphenyl-2-picrylhydrazyl) radical scavenging activty of SMS was determined according to the method of Yoshida et $a l^{26)}$ Defined amounts of SMS was incubated with $0.1 \mathrm{~mm}$ DPPH in $2 \mathrm{ml}$ methanol $/ 0.05 \mathrm{M}$ phosphate $(1: 1 \mathrm{v} / \mathrm{v})$ buffer (pH 7.6) for $10 \mathrm{~min}$ at room temperature. The kinetic decrease in DPPH absorbance at $520 \mathrm{~nm}$ was determined for the first $10 \mathrm{~min}$ after SMS addition. 


\section{RESULTS}

Shengmai San consists of three herbal components, Panax ginseng, Ophiopogon japonicus, and Schisandra chinensis. Although several antioxidant ingredients have been reported in ginseng and schisandra such as Ginseoside $\mathrm{Rb}_{1}, \mathrm{Rg}_{1}$, and Ro from ginseng ${ }^{27)}$ and schisanhenol and schizandrin B from schisandra, ${ }^{28)}$ it is important for the practical purpose to evaluate the antioxidant potentials of SMS as a TCM formulation itself. We first determined in vitro antioxidant activity of SMS by five different assay methods.

The superoxide and hydroxyl radical scavenging activities were determined by electron spin resonance (ESR) spectrometry using DMPO as the spin trap. Both the formation of superoxide and hydroxyl radical spin adduct, $\mathrm{DMPO}-\mathrm{OOH}$ and DMPO-OH, respectively, were inhibited by the addition of SMS in a dose-dependent manner (Figs. 1A, B). SMS was found to have stronger scavenging activity for hydroxyl radicals than superoxide radical. Since the dose responses for scavenging both radicals only showed linearity up to approx. $50 \%$ inhibition, the scavenging activity of the SMS $(0.6 \mathrm{~g}$ original herbs $/ \mathrm{ml}$ ) used for the following experiments was compared to that of Trolox as a reference antioxidant at $20 \%$ inhibitory concentration $\left(\mathrm{IC}_{20}\right)$. The $\mathrm{IC}_{20}$ of the SMS was calculated as 200 and $0.4 \mu \mathrm{M}$ Trolox equivalent, respectively, for superoxide and hydroxyl radical. The $\mathrm{IC}_{20}$ values of the SMS solution as determined by other in vitro antioxidant assays are summarized in Table 1. From the results, SMS was found to be rather specific scavenger toward hydroxyl radicals than toward other radicals.

The protective effect of the SMS preparation on cerebral oxidative damage induced by ischemia-reperfusion in rats was studied using two biochemical indicators: inhibition of TBARS formation and GPX activity loss in Fig. 2. After ischemia for $85 \mathrm{~min}$ and reperfusion for $45 \mathrm{~min}$ at room temperature, TBARS of the brain homogenate increased to $189.9 \%$ and GPX activity was dropped to $64.2 \%$ of untreated control brain, respectively. However, both change were effectively prevented in the brain of rats treated with SMS (15g original herb/kg) $2 \mathrm{~h}$ before the ischemia operation, such that the inhibitory activity of TBARS formation and the prevention of GPX activity loss were 98.8 and $94.9 \%$, respectively (Fig. 2). TBARS formation and GPX activity loss were inhibited by SMS in a dose-dependent manner up to $7.5 \mathrm{~g} / \mathrm{kg}$, and the damage was almost completely inhibited above this dose (Fig. 3). The dose dependence of the SMS induced GPX activity loss was not completely identical to that of inhibition of TBARS formation. SMS had little effect on the GPX preservation at the low concentration of $1.88 \mathrm{~g} / \mathrm{kg}$. However, GPX activity loss was also prevented dose-dependently with higher dose of SMS (at $3.75,7.5 \mathrm{~g} / \mathrm{kg}$ ), and the effect was saturated at $15 \mathrm{~g} / \mathrm{kg}$ as same as TBARS formation.

The protective effect of SMS was also demonstrated histochemically using the TTC staining method. TTC is reduced by mitochondrial dehydrogenases to form red hormazan pigment. The results clearly showed that SMS $(15 \mathrm{~g} / \mathrm{kg})$ administered $2 \mathrm{~h}$ before ischemia-reperfusion protected against oxidative damage in the brain (Fig. 4). A large infarcted area observed mainly in the gray matter and the brain stem remained unstained in the damaged brain of vehicle treated rats, whereas the infarction was markedly smaller in the brain

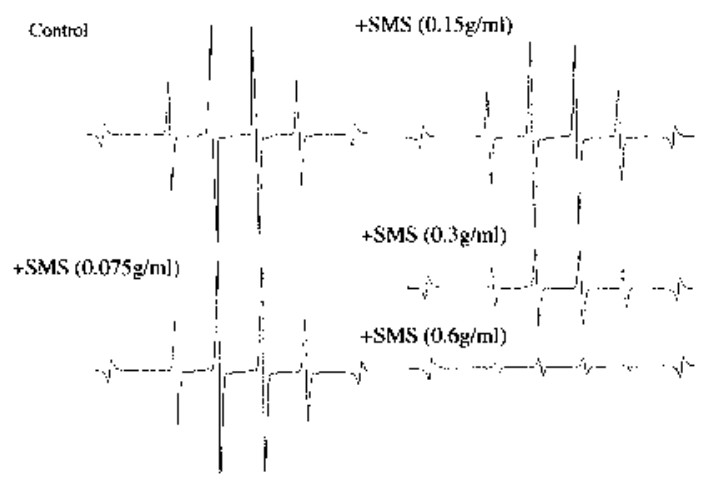

Fig. 1A. Effect of Shengmai San on DMPO-OH Formation in Vitro

Hydroxyl radicals were generated by the Fenton reaction and determined by ESR using DMPO as the spin trap. DMPO-OH signals were recorded exactly at 1 min after the addition of $\mathrm{FeSO}_{4}$ to $10 \mathrm{mM} \mathrm{H}_{2} \mathrm{O}_{2}$ solution with presence or absence of SMS. Other experimental conditions are given in the Materials and Methods.

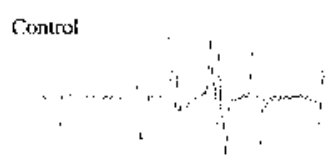

+SMS (0.15g/ml)

$+5 M S(0.075 \mathrm{~g} / \mathrm{ml})$

+ SMS (0.3-3ingl)
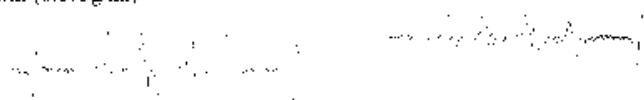

$+\operatorname{SMS}(0.6 \mathrm{~g} / \mathrm{ml})$

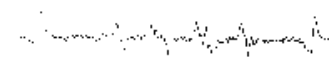

Fig. 1B. Effect of Shengmai San on DMPO-OOH Formation in Vitro

Superoxide radicals were generated by the hypoxanthine (HPX)/xanthine oxidase (XOD) system and determined by ESR using DMPO as the spin trap. DMPO-OOH signals were recorded exactly at $1 \mathrm{~min}$ after the addition of XOD with presence or absence of SMS. Other experimental conditions are given in the Materials and Methods.

Table 1. Antioxidant Activity of SMS Compared to That of Trolox as a Reference Antioxidant in Several Assay Systems in Vitro

\begin{tabular}{lc}
\hline \multicolumn{1}{c}{ Antioxidant assays } & $\mathrm{SMS} \mathrm{IC}_{20}$ as Trolox equivalent $(\mu \mathrm{M})$ \\
\hline TBARS formation & 5 \\
DPPH Quenching & 3.2 \\
Crocin bleaching test & 1.1 \\
Superoxide radical scavenging & 200 \\
Hydroxy radical scavenging & 0.4
\end{tabular}

The antioxidant potential of various concentrations of SMS and Trolox were determined in vitro by crocin bleaching, TBARS formation, DPPH quenching, and hydroxyl and superoxide radical scavenging activities, and then their $\mathrm{IC}_{20}$ determined from the dose-response curves.
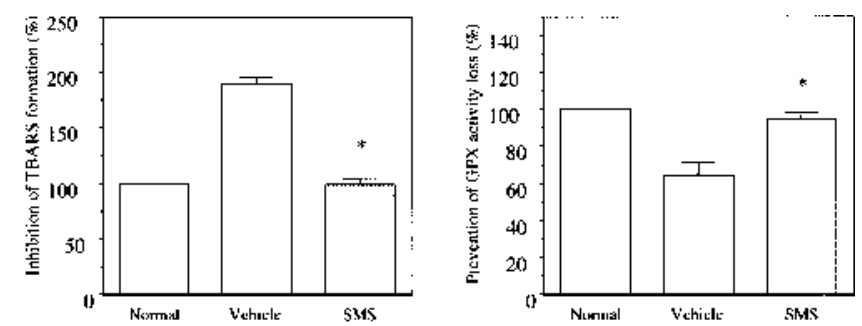

Fig. 2. Prevention of Ischemia-Reperfusion Damage by SMS in Rat Brain Inhibitory effects of SMS are given on (a) TBARS formation and (b) GPX activity loss in rat brain after ischemia-reperfusion. SMS $(15 \mathrm{~g} / \mathrm{kg})$ was administered into rat duodenum $2 \mathrm{~h}$ before ischemia induced by bilateral carotid artery occlusion for $85 \mathrm{~min}$ followed by $45 \mathrm{~min}$ reperfusion. Triplicate determinations were done or the brain homogenate prepared from each rat. Data represent the mean of 3 rats \pm S.D. $* p<0.005$, compared to Neusilin (vehicle) control. 
of SMS treated rats.

To further examine how the in vitro antioxidant potential of SMS preparation relates to the in vivo effectiveness in preventing oxidative brain damage, the antioxidant activities of SMS preparations used for Fig. 3 experiments were measured in vitro using five distinct assay systems, namely TBARS formation, DPPH quenching, crocin bleaching, and hydroxyl and superoxide radical scavenging activities. Then the in vitro antioxidant potentials were correlated with the in vivo protective activity (Figs. 5, 6).

Essentially, the SMS solutions showing stronger net in vitro antioxidant activity more effectively inhibited TBARS formation in the brain in all the in vitro antioxidant indices except TBARS inhibitory activity (Fig. 5). Of the in vitro antioxidant indices, DPPH quenching, and superoxide radical scavenging activities showed rather good correlation with inhibition of TBARS formation in vivo $\left(r^{2}=0.998,0.974\right.$, respectively.) although a linear dose-response was only established in the narrow range of both in vitro antioxidant indices. For example, the in vitro DPPH quenching activity showed linearity with the in vivo inhibition of TBARS formation only up to its $30 \%$ inhibition level, because the in vivo protection was almost completely attained with the SMS
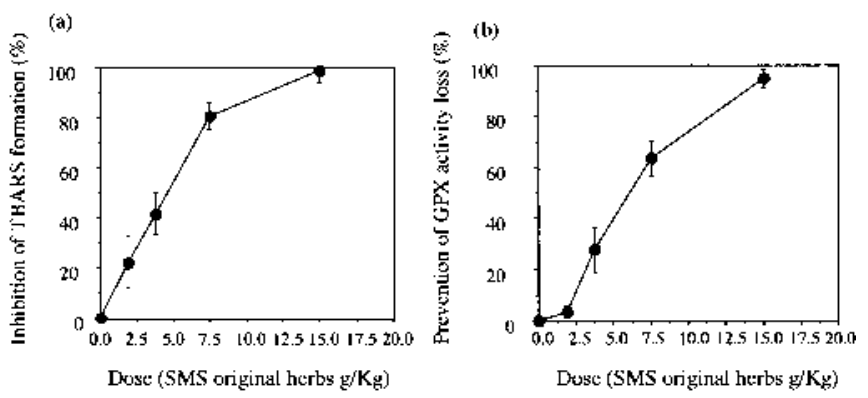

Fig. 3. Prevention of Ischemia-Reperfusion Damage by SMS in Rat Brain Inhibitory effects of SMS are given for (a) TBARS formation and (b) GPX activity loss in rat brain after ischemia-reperfusion. SMS $(1.88,3.75,7.5,15 \mathrm{~g} / \mathrm{kg})$ was administered into rat duodenum $2 \mathrm{~h}$ before ischemia induced by bilateral carotid artery occlusion for $85 \mathrm{~min}$ followed by $45 \mathrm{~min}$ reperfusion. Triplicate determinations were done for the brain homogenate prepared from each rat. Data represent the mean of 3 the rats \pm S.D. giving approx. $30 \%$ inhibition of DPPH quenching in vitro. The same trends were observed for the in vitro hydroxyl radical and superoxide radical scavenging activities, i.e., linear correlation was only observed up to approx. 20 and $40 \%$ inhibition levels of both activities in vitro, respectively. On the other hand, the in vitro crocin bleaching assay covered the in vivo TBARS inhibitory activity with its rather wide range of activity, although their relationship was not completely linear.

The same correlation patterns were observed between the in vitro antioxidant indices and the in vivo protective effect of SMS as defined by GPX activity loss (Fig. 6). Only limited activity ranges of the in vitro indices (for example, in DPPH quenching and superoxide radical scavenging, up to approx. 30 and $60 \%$ inhibition levels, respectively) covered the whole range of the in vivo effect $(0-100 \%)$ on protecting against GPX activity loss in all the in vitro assays except the crocin bleaching assay.

\section{DISCUSSION}

In the present study, we examined the protective effect of SMS against cerebral oxidative injury as a model of antioxidant-based composite therapy for oxidative stress-related diseases. Not only biochemical indicators but also histochemical observation clearly showed that SMS, a hydroxyl radical scavenging TCM, effectively prevented the cerebral oxidative damage induced by forebrain ischemia followed by reperfusion. The dose-response profile of the SMS effect was almost the same as those obtained for single antioxidant drug, when a granulated SMS decoction was used.

For therapeutic purposes, TCM is usually administered orally, but oral administration was found not suitable for the present experiment that relatively acute effect of SMS was investigated. We found the SMS administered directly into the rat duodenum gave stable and reproducible in vivo results.

The dose dependence of the SMS effect was not completely identical for the inhibition of TBARS formation and GPX activity loss in vivo, especially at the low SMS concen-

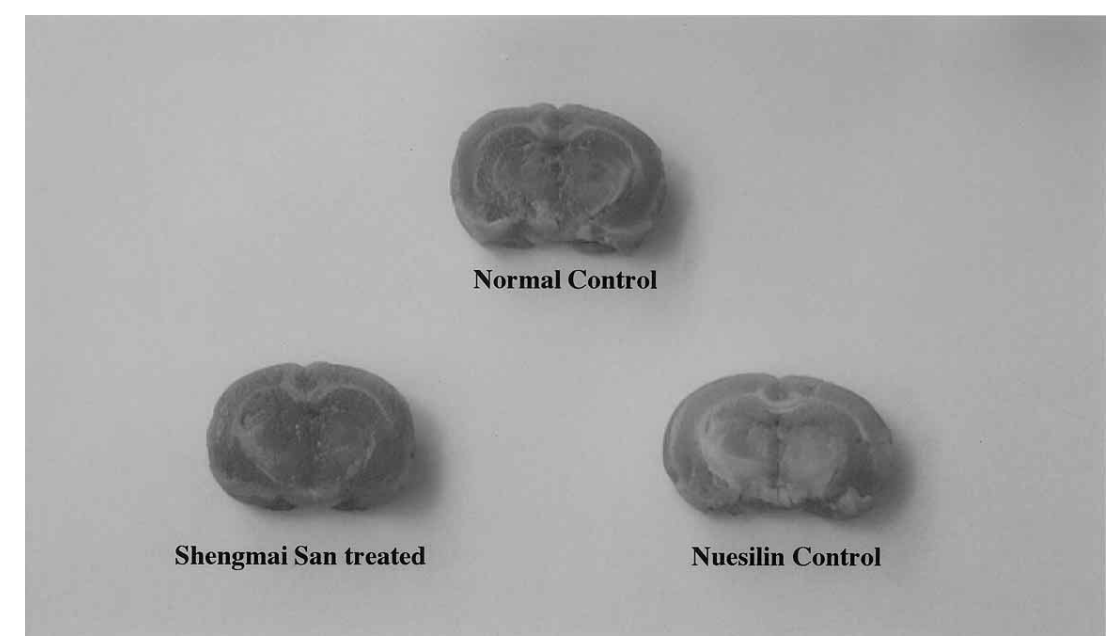

Fig. 4. Protective Effect of Shengmai San against Cerebral Ischemia-Reperfusion Damage in Rat Evaluated by TTC Staining

Brain coronary sections were prepared after $85 \mathrm{~min}$ ischemia followed by $45 \mathrm{~min}$ reperfusion, and then stained with 2,3,5-triphenyltetrazolium. Normal control; rats not given any treatment, Neusilin control; rats administered Neusilin instead of SMS before ischemia followed by reperfusion, SMS treated; rats treated with SMS before cerebral ischemiareperfusion. Each group contained 3 rats, and the results for the other two rats in each group were almost identical to those represented here. 

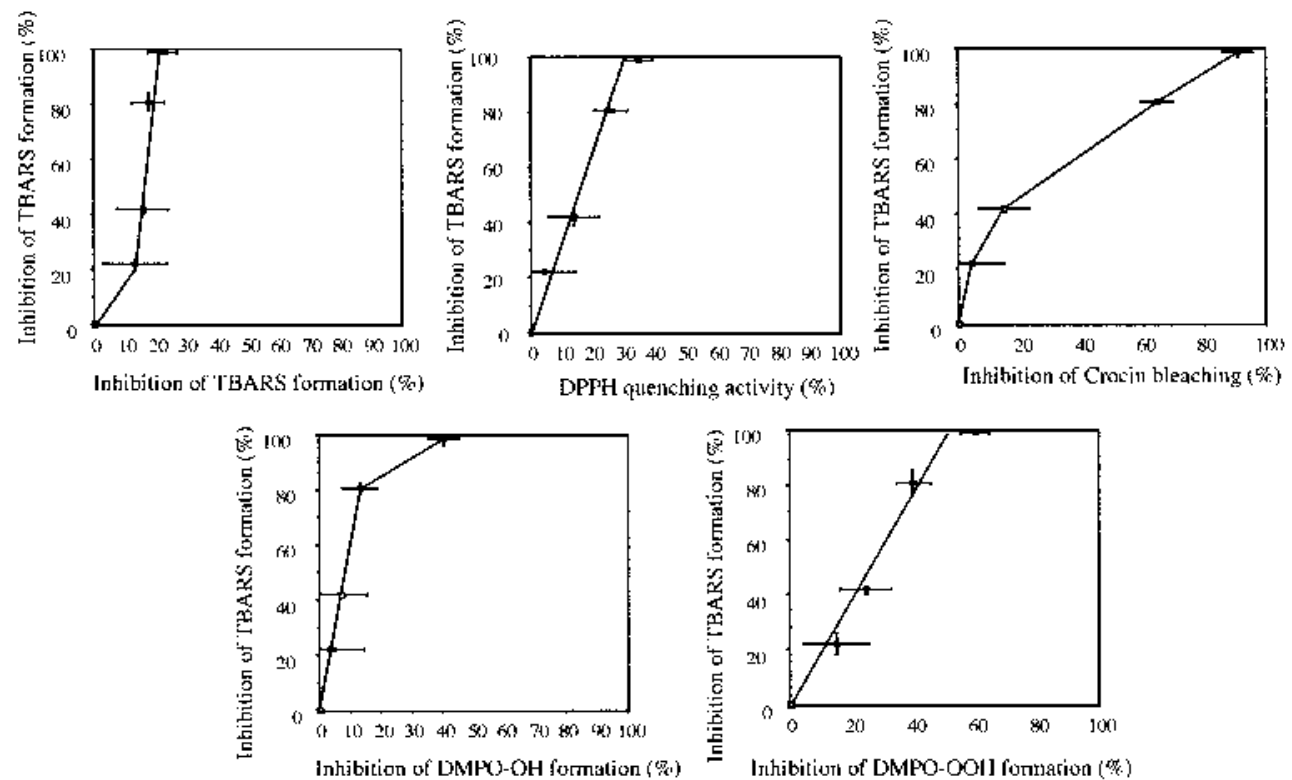

Fig. 5. Comparison of in Vivo and in Vitro Antioxidant Activities of SMS

Antioxidant potentials of SMS solutions of different concentration were determined by five different in vitro assays. The in vitro antioxidant potential was determined and then correlation to the inhibitory activities on TBARS formation in vivo obtained by the experiments in Fig. 3. Data represent the mean \pm S.D. of 3 independent in vivo and in vitro experiments.
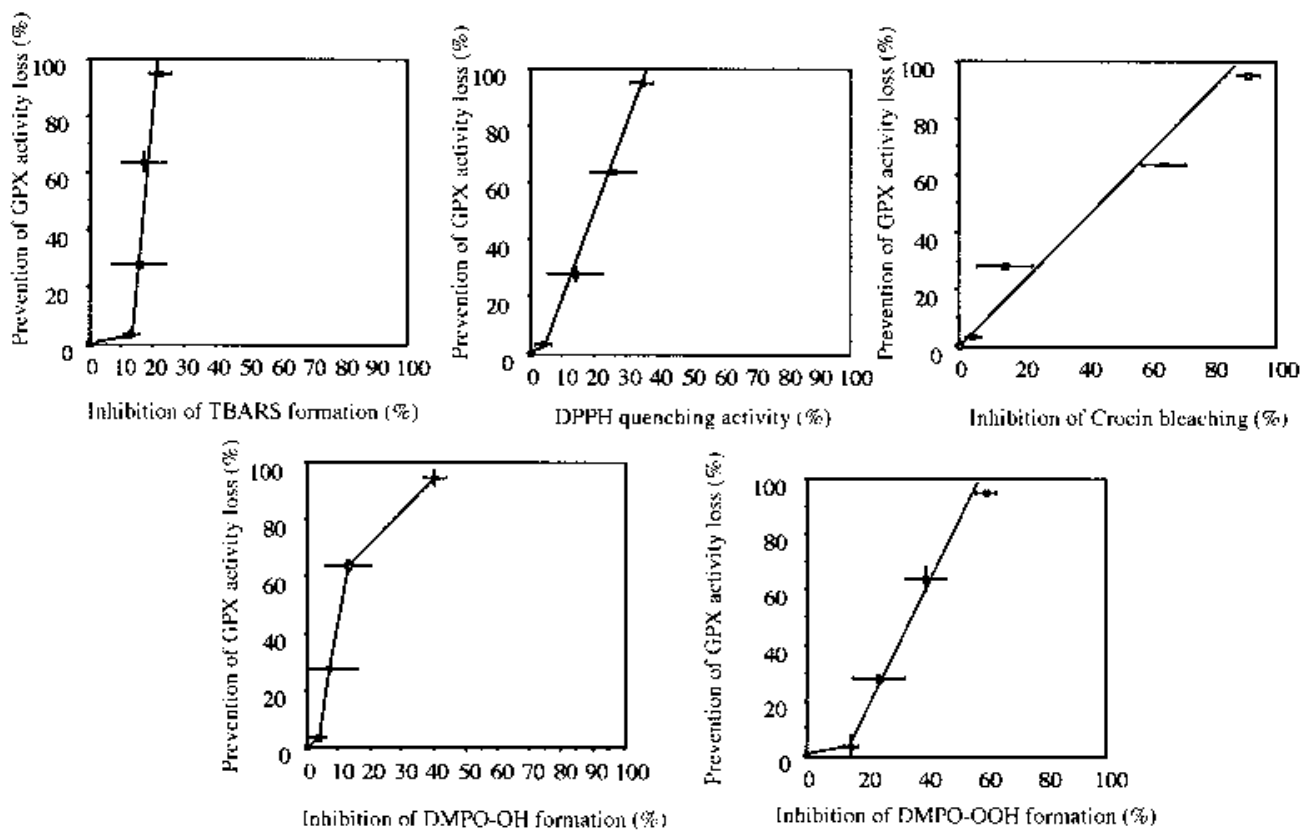

Fig. 6. Comparison of in Vivo and in Vitro Antioxidant Activities of SMS

The in vitro antioxidant potentials at different concentration of SMS solution as above were assessed for correlation with the preventive effect on GPX activity loss in vivo as in Fig. 3.

tration (see Figs. 3a,b). Since GPX activity change reflects more likely the cellular physiological response, this differential effect of SMS on these two biochemical indices suggests that the SMS not only functions as a simple antioxidant to inhibit lipid peroxidation but also as a modulator of the cellular antioxidant potential by that antioxidant enzymes such as GPX was enhanced. Indeed, we recently found that SMS enhanced cellular GPX levels in cultured cells (unpublished data). Therefor, TCM is a reliable model for studying antioxidant-based composite therapy of the oxidative stress-related disorders, although further studies are required to identify the specific agent participating in each biochemical steps for the brain damage protection.

Since oxidative stress is implicated ubiquitously in age and life-style related pathophysiology such as diabetes miluitus, cancer and dementia to that TCM is applicable, it is important to know how the in vitro antioxidant potential of TCM itself as a mixed formula correlates to the in vivo therapeutic effectiveness. However, it is still argued that the in vitro antioxidant potential determined by certain assay method is not 
always consistent with those determined by other assay methods, and also, the in vitro antioxidant potential is not sufficiently reflected in the in vivo effectiveness. Therefore, we here again examined precisely the in vitro antioxidant potential of different SMS preparations by five distinct typical assays of antioxidants in vitro and then each activity determined by different methods was correlated to the in vivo effectiveness of the same SMS preparation in preventing oxidative brain damage produced by ischemia reperfusion in rats.

Results showed that all of the in vitro antioxidant indices essentially reflect the in vivo activity in preventing cerebral oxidative damage, that is, both in the inhibition of TBARS formation and the prevention of GPX activity loss. However, each in vitro antioxidant index differently correlated to the in vivo activity as was expected from the observation in Table 1 that each antioxidant assay gives different $\mathrm{IC}_{20}$ for SMS. Moreover, crocin bleaching test covered whole range of the in vivo effect although the relation was not sufficiently linear, but in other indices, the linearity between the in vitro and in vivo activity was established only for a limited region of the observed in vitro activities, especially for TBARS inhibition and hydroxyl radical scavenging activity. It was thus suggested that TBARS inhibition and hydroxyl radical scavenging activities in vitro are not adequate index for predicting the in vivo effectiveness of different SMS solutions. This might be due to the reason that the linear dose-response is hardly established even for the in vitro antioxidant assays. For example, apparent hydroxyl radical scavenging activity is usually saturated at around 50\% inhibition level even with increasing concentrations of test samples when determined by ESR using Fenton system as a radical generator (unpublished observation). It was also noted that the in vivo prevention was almost completely attained even with the SMS preparations showing rather low antioxidant activity in vitro (see Fig. $3)$.

Present study suggested that inhibitory activity of crocin bleaching and superoxide scavenging activity are rather reasonable index for the evaluation of the in vivo antioxidant potential of mixed formula like TCM in vivo. Moreover, attention must be paid to the activity range of the in vitro antioxidant index that is applicable for the quantitative estimation of the in vivo antioxidant potential of certain mixed formula.
Acknowledgements We thank Kotaro Kampo Pharmaceutics Co. Ltd. for preparing the granulated SMS. This study was supported by a grant from the Promotion and Mutual Aid Corporation for Private schools of Japan.

\section{REFERENCES}

1) Coyle J. T., Puttfarcken P., Science., 262, 689-695 (1993).

2) Liu J., Mori A., Neurochemical Res., 24, 1479-1497 (1999).

3) Weisbrot-Lefkowitz M., Reuhl K., Perry B., Chan P. H., Inoue M., Mirochnitchenko O., Mol. Brain. Res., 53, 333-338 (1998).

4) Halliwell B., J. Neurochemistry., 59, 1609-1623 (1992).

5) Tibor K., Bo K. S., Stroke., 29, 705-718 (1998).

6) Kim Y. C., Markelonis G. J., Oh T. H., J. Neurosci. Res., 53, 426-432 (1998).

7) Muller D. P. R., Goss-Sampson M. A., Crit. Rev. Neurobiol., 5, 239265 (1990).

8) Muller R., Cadenas R., Graf P., Sies H., Biochem. Pharmacol., 33, 3235-3240 (1984).

9) Hall E. D., Pazara K. E., Braughler J. M., Linemon K. L., Jacobsen E. J., Stroke., 21, 83-87 (1990).

10) Halliwell B., Gutteridge J. M. C., Lancet., I, 1396-1398 (1984).

11) Halliwell B., Gutteridge J. M. C., Biochem. J., 219, 1-14 (1984).

12) Behl C., Int. J. Vitam. Nutr. Res., 69, 213-219 (1999).

13) Floyd R. A., Proc. Soc. Exp. Biol. Med., 222, 236-245 (1999).

14) Gong X., Sucher N. J., Trends. Pharmacol. Sci., 20, 191-196 (1999).

15) Cheng J. T., J. Clin. Pharmacol., 40, 445-450 (2000).

16) Oishi M., Mochizuki Y., Takasu T., Chao E., Nakamura S., Alzheimer. Dis. Assoc. Disord., 12, 247-250 (1998).

17) Shin H.-C., Chang H.-K., Chen F.-L., Chen C.-M., Chen S.-C., Lin Y.T., Shibuya T., Am. J. Chin. Med., 28, 77-86 (2000).

18) Wang X.-J., Magara T., Konishi T., Free Rad. Res., 31, 449-455 (1999).

19) Wang X.-J., Ichikawa H., Konishi T., Biol. Pharm. Bull., 24, 558-563 (2001).

20) Isayama K., Pitts L. H., Nishiyama M. C., Stroke., 22, 1394-1398 (1991).

21) Ohkawa H., Ohishi N., Yagi K., Anal. Biochem., 95, 351-358 (1979).

22) Albrecht W., Methods Enzymol., 77, 325-333 (1981).

23) Ali M. A., Konishi T., Biochem. Mol. Biol. In., 46, 137-145 (1998).

24) Finkelstein E., Rosen G. M., Rauckman E. J., Arch. Biochem. Biophys., 200, 1-16 (1980).

25) Wolf B., Christa M., Manfred S., Biochem. Biophys. Acta, 796, 312 319 (1984).

26) Yoshida T., Mori K., Hatano T., Okumura T., Uehara I., Komagoe K., Fujita Y., Okuda T., Chem. Pharm. Bull., 37, 1919-1921 (1989).

27) Wen T.-C., Yoshimura H., Matsuda S., Lim J.-H., Sakanaka M., Acta Neutropathol, 91, 15-22 (1996).

28) Xue J.-Y., Liu G.-T., Wei H.-L., Pan Y., Free. Radical. Biol. Med., 12, $127-135$ (1992). 